\title{
SOME MIXED MATRIX PROBLEMS OVER SEVERAL DISCRETE VALUATION RINGS
}

\author{
Nadiya Gubareni \\ Institute of Mathematics, Czestochowa University of Technology \\ Czestochowa, Poland \\ nadiya.gubareni@yahoo.pl
}

\begin{abstract}
This article presents some results about several district valuation rings with a common skew field of fractions. They are obtained from the approximation theorem for discrete valuation rings. These results give the possibility to solve basic mixed matrix problems for such rings. We present the solution of some mixed flat matrix problems over several district valuation rings with common skew field of fractions.
\end{abstract}

Keywords: valuation rings, local invariant rings, discrete valuation rings, approximation theorem, mixed flat matrix problems

\section{Introduction}

In this paper we present the solution of some basic mixed flat matrix problems over several discrete valuation rings with a common skew field of fractions.

Let $O$ be a discrete valuation ring with a skew field of fractions $D$. Recall that a right elementary row operation over $D$ in an arbitrary matrix is one of the following:

(i) interchanging of two rows;

(ii) multiplying a row by a nonzero scalar (i.e. an element of $D$ );

(iii) replacing a row by itself plus a scalar multiple of another row.

Analogously there can be introduced the left $D$-elementary column operations. In a similar fashion we can introduce right $O$-elementary row transformations and left $O$-elementary column transformations.

Let $\left\{O_{i}\right\}$ be a family of discrete valuation rings (not necessary commutative) with Jacobson radicals $R_{i}(i=1,2, \ldots, k)$ and a common skew field of fractions $D$.

Assume that $A$ is an indecomposable semiperfect ring with a decomposition of the identity into a sum of pairwise orthogonal idempotents

$$
1=f_{1}+f_{2}+\ldots+f_{n}
$$

such that the two-sided Pierce decomposition of $A$ has the following form: 


$$
A=\bigoplus_{i, j=1}^{n} f_{i} A f_{j}
$$

where $f_{i} A f_{i}=H_{n_{i}}\left(O_{i}\right)$ for $i=1,2, \ldots, k ; f_{j} A f_{j}=D$ for $j=k+1, \ldots, n$; and $A_{i j}=f_{i} A f_{j}$ is an $\left(A_{i i}, A_{j j}\right)$-bimodule, for $i, j=1,2, \ldots, n$.

Consider the main flat matrix problem associated with a ring A given by (2) of the following form.

\section{Main mixed flat matrix problem}

Given a block matrix $\mathbf{T}$ :

\begin{tabular}{|c|c|c|c|c|}
\hline $\mathbf{T}_{11}$ & $\ldots$ & $\mathbf{T}_{1 j}$ & $\ldots$ & $\mathbf{T}_{1 n}$ \\
\hline$\ldots$ & $\ldots$ & $\ldots$ & $\ldots$ & $\ldots$ \\
\hline $\mathbf{T}_{i 1}$ & $\ldots$ & $\mathbf{T}_{i j}$ & $\ldots$ & $\mathbf{T}_{i n}$ \\
\hline$\ldots$ & $\ldots$ & $\ldots$ & $\ldots$ & $\ldots$ \\
\hline $\mathbf{T}_{n 1}$ & $\ldots$ & $\mathbf{T}_{n j}$ & $\ldots$ & $\mathbf{T}_{n n}$ \\
\hline
\end{tabular}

partitioned into $n$ horizontal and vertical strips so that a block $\mathbf{T}_{i j}$ has elements in $f_{j} A f_{i}$ and $\mathbf{T}_{i i}$ has elements in $f_{i} R f_{i}$ for $i, j=1, \ldots, n$.

Reduce the matrix $\mathbf{T}$ to a block-diagonal form with indecomposable blocks by means of admissible transformations $\mathbf{T} \rightarrow \mathbf{U T V}$, where $\mathbf{U}=\left(\mathbf{U}_{i j}\right), \mathbf{V}=\left(\mathbf{V}_{i j}\right)$ are block unsingular matrices, and $\mathbf{U}_{i i}, \mathbf{V}_{i i}$ are square invertible matrices with entries in $f_{i} A f_{i}(i=1,2, \ldots, n)$.

Definition 1.1. The vector of the form:

$$
\mathbf{d}=\mathrm{d}(\mathbf{T})=\left(d_{1}, d_{2}, \ldots, d_{n} ; d^{1}, d^{2}, \ldots, d^{n}\right),
$$

where $d_{i}$ is the number of rows of the $i$-th horizontal strip of $\mathbf{T}$, and $d^{i}$ is the number of columns of the $i$-th vertical strip of $\mathbf{T}$ for $i=1,2, \ldots, n$ is called the dimension vector of a stripped matrix $\mathbf{T}$

$$
\operatorname{dim}(\mathbf{T})=\sum_{j=1}^{n} d_{j}+\sum_{i=1}^{n} d^{i}
$$

Definition 1.2. We say that a mixed flat matrix problem with a matrix $\mathbf{T}$ is of bounded representation type if there is a constant $C$ such that $\operatorname{dim}(\mathbf{T})<C$ for all the indecomposable matrices $\mathbf{T}$. Otherwise it is of the unbounded representation type. 
The solution of a mixed flat matrix problem in the case of one discrete valuation ring and its skew field of fractions was considered by A.G. Zavadskij and U.S. Revitskaya in [1]. Earlier similar problems were studied in [2-5].

We now describe briefly the contents of this paper. In Section 2 we present the main notions and results concerning valuations and structures of (noncommutative) discrete valuations rings (DVRs). Section 3 considers the approximation theorem for DVRs and presents some important corollaries which makes it possibile to solve basic mixed flat matrix problems. Using these results in Section 4 we give the solutions of some basic mixed flat matrix problems over several distinct discrete valuation rings and their common skew field of fractions. These matrix problems arise in connection with problems in the representation theory of rings.

We use the main notions and results from [6,7].

All rings considered in this paper are assumed to be associative with $1 \neq 0$ and all modules are assumed to be unital.

\section{Valuations and noncommutative DVRs}

In this section we recall some main notions and results from the theory of valuations for noncommutative rings. First noncommutative valuation rings were considered and studied by O.F.G. Schilling in [8]. Below we give the main notions of a valuation and a valuation ring of a division ring. They are the generalizations of the concepts for commutative case which were first introduced and studied in 1932 by W. Krull [9].

Definition 2.1. [8] Let $G$ be a totally ordered group (written additively) with order relation $\geq$. Add to $G$ a special symbol $\infty$ such that $x+\infty=\infty+x=\infty$ for all $x \in G$. Let $D$ be a division ring. A valuation on $D$ is a surjective map $v: D \rightarrow G \cup \infty$ which satisfies the following:

1) $v(x) \leq \infty$

2) $v(x)=\infty$ if and only if $x=0$

3) $v(x y)=v(x)+v(y)$

4) $v(x+y) \geq \min (v(x), v(y))$

for all $x, y \in D$.

Definition 2.2. [8] A subring $R$ of a division ring $D$ is called the valuation ring (=non-commutative valuation ring) of $D$ if there is a totally ordered group $G$ and a valuation $v: D \rightarrow G \cup \infty$ of $D$ such that

$$
\mathrm{R}=\{x \in D: v(x) \geq 0\}
$$

In his paper [8] O.F.G. Schilling introduced the important notions of invariant valuation rings and total valuations rings and systematically studied them. 
Definition 2.3. [8] A subring $R$ of a division ring $D$ is called invariant of $D^{*}$ if $d R d^{-1}=R$ for any $d \in D^{*}$. A subring $R$ of a division ring $D$ is called a total valuation ring if $d^{-1} \in R$ for any $d \notin R$.

The next theorem shows that the notions of valuation rings of division rings and total valuation rings are equivalent.

Theorem 2.4. [8] Let $R$ be a subring of a division ring $D$. Then the FCAE:

1) $R$ is a valuation ring of $D$ with respect to some valuation $v$ on $R$;

2) $R$ is invariant total valuation ring of $D$.

Similar to the commutative case we can introduce the notion of discrete valuation rings for noncommutative case. In the literature it was defined by many different ways. Below we give the definition of DVR following to O.F.G. Schilling.

Definition 2.5. [8] A subring $O$ of a division ring $D$ is called the (noncommutative) discrete valuation ring (DVR for short) if there is a (discrete) valuation $v: D \rightarrow \mathbf{Z}$ of $D$ such that

$$
O=\{x \in D: v(x) \geq 0\} .
$$

The basic properties of DVR's are presented in the following proposition.

Proposition 2.6. [10] Let $O$ be a DVR of a division ring $D$ with respect to a valuation $v$. Let $t$ be a fixed element of $O$ with $v(t)=1$. Then

1) $O$ is a local domain with the nonzero unique maximal ideal

$$
M=\{x \in O: v(x)>0\}
$$

2) Any nonzero element $x \in O$ has the unique representation in the form

$$
x=t^{n} u=w t^{n}
$$

for some $u, w \in O^{*}$, and $n \in \mathbf{Z}, n \geq 0$.

If $D$ is a division ring of fractions of $A$ then any element $y \in D^{*}$ has the form $y=t^{n} u=w t^{n}$ for some $u, w \in O^{*}$, and $n \in \mathbf{Z}$.

3) Any one-sided ideal $I$ of $O$ is a two-sided ideal and has the form $I=t^{n} O=O t^{n}$ for some $n \in \mathbf{Z}, n \geq 0$, i.e. $O$ is a principal ideal ring. In particular, $M=t O=O t$, and $I=M^{n}=t^{n} O=O t^{n}$.

4) $\bigcap_{i=0}^{\infty} M^{i}=0$, where $M$ is the unique maximal ideal of $O$.

5) $O$ is a Noetherian uniserial ring.

6) $O$ is a hereditary ring.

As we mentioned above there were a few different definitions of DVRs. The following statement shows that all these definitions of DVRs are equivalent. 
Proposition 2.7. [10] The following statements for a ring $O$ are equivalent.

1) $O$ is a DVR.

2) $O$ is a local ring with nonzero maximal ideal $M$ of the form $M=t O=O t$, where $t \in O$ is a non-nilpotent element, and $\bigcap_{i=0}^{\infty} M^{i}=0$.

3) $O$ is a local principal ideal domain which is not a division ring.

4) $O$ is a Noetherian local ring with nonzero maximal ideal which is two-sided and principal.

5) $O$ is a right (left) Noetherian local ring with nonzero maximal ideal $M$ of the form $M=t O=O t$ with a non-nilpotent element $t \in O$.

6) $O$ is a Noetherian non-Artinian uniserial ring.

7) $O$ is a Noetherian valuation ring.

\section{Approximation theorem for DVR}

The approximation theorem is a basic tool for working with several valuation rings over a given skew field. In this section we consider the approximation theorem for noncommutative DVRs and obtain some corollaries from this theorem. In [11] H.H. Brungs and J. Gräter introduced the following general notion of a valuation on a skew field:

Definition 3.1. [11] Let $D$ be a skew field and $W_{v}$ a totally ordered set with largest element $\theta$. A mapping $v: D \rightarrow W_{v}$ is called a valuation of $D$ if for all $x, y, z \in D$ the following conditions hold:

(i) $v(x)=\theta$ if and only if $x=0$;

(ii) $v(x+y) \geq \min \{v(x), v(y)\}$;

(iii) $v(x) \geq v(y)$ implies $v(z x) \geq v(z y)$.

This valuation defines the subring

$$
B_{v}=\{x \in D: v(x) \geq v(1)\}
$$

which is a total ring in the sense that $x \notin B_{v}$ implies $x^{-1} \in B_{v}$ for each $x \in D$.

Note that if in the definition above the condition iii) is changed by (iv) $v(x y)=v(x)+v(y)$

provided $W_{v} \mid \theta=G$ is a totally ordered Abelian group (written additively) we obtain the definition of a valuation which was first proposed by Schilling in [8].

J. Gräter in [12] introduced the important notion of a locally invariant ring.

Definition 3.2. [12] A ring $R$ is called right locally invariant if for each right ideal $I$ of $R$ 


$$
\sqrt{I}=\left\{x \in R \mid \exists n_{x} \in N, x^{n_{x}} \in I\right\}
$$

is a completely prime ideal.

The following theorem gives the characterization of locally invariant valuation rings.

Theorem 3.3. [13, Theorem 3.1] A chain domain $R$ is locally invariant if and only if any one of the following equivalent conditions is satisfied:

1) $\cap a^{n} R$ is a two-sided ideal for any $a \in R$.

2) $R a^{2} \subseteq a R$ for any $a \in R$.

3) $\sqrt{I}=\left\{x \in R \mid \exists n_{x} \in N, x^{n_{x}} \in I\right\}$ is a completely prime ideal for every right ideal $I$ in $R$.

4) Between any two prime ideals of $R$ there is another two-sided ideal of $R$.

5) $x P(x)=P(x) x$ for any $x \in R$ and $P(x)$ the minimal completely prime ideal of $R$ containing $x$.

From this theorem it immediately follows the following result:

Corollary 3.4. Any discrete valuation ring $O$ of a division ring $D$ (in sense of Definition 2.5) is locally invariant.

In [12] J. Gräter proved the approximation theorem for total valuation rings of a skew field when these rings are locally invariant. Here we give this theorem following to [13]. We need some definitions which were introduced in this paper.

Let $B_{1}, B_{2}$ be valuation rings of a skew field $D$. Then there exists a minimal valuation ring $B_{1,2}$ of $D$ containing both $B_{1}$ and $B_{2}$. The right ideal $I_{1}$ of $B_{1}$ and the right ideal $I_{2}$ of $B_{2}$ are compatible if $I_{1} B_{1,2}=I_{2} B_{1,2}$. If in addition, $a_{1}, a_{2} \in D$ then $a_{1}, a_{2}, I_{1}, I_{2}$ are called compatible if $a_{1}-a_{2} \in I_{1} B_{1,2}=I_{2} B_{1,2}$.

Theorem 3.5. [13] (Approximation Theorem).

Let $B_{1}, \ldots, B_{n}$ be valuation rings of a skew field $D$ and assume that $B_{i}$ is locally invariant for $i=1, \ldots, n-1$. Let $a_{\mathrm{i}} \in D$ for $i=1, \ldots, n$ and let $I_{i}$ be a right ideal of $B_{i}$ such that $a_{i}, a_{j}, I_{i}, I_{j}$ are compatible for every $i, j \in\{1, \ldots, n\}$. Then there exists an $x \in D$ with

$$
x-a_{i} \in I_{i}
$$

for $i=1, \ldots, n$.

This theorem holds for valuation rings in finite dimensional division algebras.

Let $B_{1}, B_{2}$ be discrete valuation rings of $D$. Since any discrete valuation ring $O$ of $D$ is a maximal valuation ring in $D$, then $B_{1,2}=D$. Therefore $I_{1} B_{1,2}=I_{2} B_{1,2}$ for 
each ideal $I_{1}$ of $B_{1}$ and each ideal $I_{2}$ of $B_{2}$, and so $a_{1}, a_{2}, I_{1}, I_{2}$ are compatible for any elements $a_{1}, a_{2} \in D$ and any ideal $I_{1}$ of $B_{1}$, any ideal $I_{2}$ of $B_{2}$.

Taking into account that any discrete valuation ring is locally invariant we immediately obtain the following corollary from Theorem 3.5, which is an approximation theorem for discrete valuation rings.

Corollary 3.6. (Approximation Theorem for DVRs) Let $O_{1}, \ldots, O_{n}$ be distinct discrete valuation rings of a skew field of fractions $D$. Then for any elements $a_{i} \in D$ and ideals $I_{i}$ of $O_{i}$ for $i=1, \ldots, n$ there is an element $x \in D$ such that

$$
x-a_{i} \in I_{i}
$$

for $i=1, \ldots, n$.

Proposition 3.7. Let $O_{1}, O_{2}$ be distinct discrete valuation rings in a skew field of fractions $D$. Then

1. $D=O_{1}+O_{2}$.

2. There exist elements $\pi_{1} \in M_{1} \backslash M_{1}^{2}$ and $\pi_{1} \in O_{2}{ }^{*}$. Analogously there exist elements $\pi_{2} \in M_{2} \backslash M_{2}^{2}$ and $\pi_{2} \in O_{1}{ }^{*}$.

3. $D^{*}=O_{1}^{*} \cdot O_{2}^{*}$.

Proof.

1. Put $I_{1}=O_{1}, I_{2}=O_{2}, a_{1}=d \in D, a_{2}=0$ in Corollary 3.6. Then there exist elements $x \in D$ that $x-d \in O_{1}$ and $x \in O_{2}$, i.e. $x-d=a, x=b$. So $d=b-a$, where $b \in O_{2}, a \in O_{1}$.

2. Put $I_{1}=M_{1}^{2}, I_{2}=M_{2}, a_{1} \in M_{1} \backslash M_{1}^{2}, a_{2}=1$ in Corollary 3.6.

3. This follows immediately from Theorem 2.6 (3).

Proposition 3.8. Let $O_{1}, O_{2}$ be distinct discrete valuation rings in a skew field of fractions $D$ and $\mathrm{A}=O_{1} \cap O_{2}$. Then there exists an element $\mathrm{x} \in D$ such that $x^{n} \notin A$ and $x^{-n} \notin A$ for any $n \in \mathbf{N}$.

Proof. Suppose that $A$ is a total valuation ring of $D$. Then by [14, Corollary 1.3] subrings of $D$ containing $A$ must be totally ordered by inclusion. Since $O_{1} \not \subset O_{2}$ and $O_{2} \not \subset O_{1}$ we have a contradiction.

Therefore $A$ is not a total valuation ring and so by definition there exists and element $x \in D$ such that $x \notin A$ and $x^{-1} \notin A$.

Suppose there exists $n \in \mathbf{N}$ such that $x^{n} \in A$ or $x^{-n} \in A$. Since $x^{n} \in A, x^{n} \in O_{1}$ and $x^{n} \in O_{2}$. Therefore $v_{1}\left(x^{n}\right)=n v_{1}(x) \geq 0$, which implies that $v_{1}(x) \geq 0$, that is $x \in O_{1}$. Analogously, $x \in O_{2}$, that is, $x \in A$. A contradiction! In the same way we can show that $x^{-n} \notin A$ for any $n \in \mathbf{N}$. 


\section{Some mixed matrix problems}

In this Section we give the solution of two mixed matrix problems. Consider the first one.

\section{Matrix problem I}

Let $O_{1}, O_{2}$ be a district valuation rings of the division ring $D$. Given a matrix $\mathbf{T}$ with entries in $D$ it is possible the following admissible transformations:

1) left $O_{1}$ - elementary transformations of rows of the matrix $\mathbf{T}$.

2) right $\mathrm{O}_{2}$ - elementary transformations of columns of the matrix $\mathbf{T}$.

Find all indecomposable matrices.

Lemma 4.1. The matrix problem I can be reduced by admissible transformations to the following form:

$$
\mathbf{T}=\left[\begin{array}{ll}
\mathbf{I} & \mathbf{O} \\
\mathbf{O} & \mathbf{O}
\end{array}\right]
$$

Proof.

1-st step.

Let $M_{i}$ be a maximal ideal of a ring $O_{\mathrm{i}}$. By Proposition 3.7 there exists an element $\pi_{1} \in M_{1} \backslash M_{1}^{2}$ and $\pi_{1} \in O_{2}{ }^{*}$. Analogously there exists an element $\pi_{2} \in M_{2} \backslash M_{2}^{2}$ and $\pi_{2} \in O_{1}^{*}$.

So that any element $x \in D$ can be written in the different forms $x=\pi_{1}{ }^{n} \varepsilon=\eta \pi_{1}{ }^{n}$ and $x=\pi_{2}{ }^{m} \mu=\xi \pi_{2}{ }^{m}$, where $\varepsilon, \eta \in O_{1}{ }^{*}$ and $\mu, \xi \in O_{1}$. Then $v_{1}(x)=n$ and $v_{2}(x)=m$.

Pick out in the matrix $\mathbf{T}$ an element $x=\pi_{2}{ }^{m} \mu \in D$ such that $v_{2}(x)$ is minimal among all elements of $\mathbf{T}$ and put it in the place $(1,1)$. Multiplying the first column of this matrix on $\mu^{-1} \in O_{2}{ }^{*}$ we obtain that $t_{11}=\pi_{2}{ }^{m}$. By this element we can make all elements in the first row to be zero using the transformations on the columns. So we obtain the equivalent form of the matrix:

$$
\mathbf{T}=\left[\begin{array}{cc}
\pi_{2}^{n} & \mathbf{O} \\
* & \mathbf{T}_{1}
\end{array}\right]
$$

Since $\pi_{2}^{n} \in O_{1}{ }^{*}$, multiplying the first row by $\pi_{2}{ }^{-n} \in O_{1}{ }^{*}$, we can reduce the matrix $\mathbf{T}$ to the following equivalent form:

$$
\mathbf{T}=\left[\begin{array}{ll}
1 & \mathbf{O} \\
* & \mathbf{T}_{1}
\end{array}\right]
$$

Applying this procedure to the matrix $\mathbf{T}_{1}$ we obtain the following equivalent form of the matrix $\mathbf{T}$ : 


$$
\mathbf{T}=\left[\begin{array}{ll}
\mathbf{T}_{2} & \mathbf{O} \\
* & \mathbf{O}
\end{array}\right] \text {, where } \mathbf{T}_{2}=\left[\begin{array}{cccc}
1 & 0 & \cdots & 0 \\
* & 1 & \ddots & 0 \\
\vdots & \ddots & \ddots & 0 \\
* & * & * & 1
\end{array}\right] .
$$

Since $O_{1}+O_{2}=D$, then for any nonzero element $x=t_{i j} \in \mathbf{T}_{2}$ with $i>j$ there exist elements $a \in O_{1}$ and $b \in O_{2}$ such that $-x=a+b$. So by transformations on the $i$-th row and the $j$-th column of $\mathbf{T}_{2}$ we can make this element to be 0 . So we reduce the matrix $\mathbf{T}$ to the following equivalent form:

$$
\mathbf{T}=\left[\begin{array}{cc}
\mathbf{I} & \mathbf{O} \\
\mathbf{T}_{3} & \mathbf{O}
\end{array}\right]
$$

where $\mathbf{I}$ is the identical matrix.

2-nd step.

Let elements of $\mathbf{T}_{3}$ have form $t_{i j}$ with $i \geq k$. We pick out in the matrix $\mathbf{T}_{3}$ an element $y \in D$ with minimal $v_{1}(y)$ for all elements in $\mathbf{T}_{3}$ and put it in the place $(k, 1)$. Let $y=\pi_{1}{ }^{n}$. If $n \geq 0$ then by the element $t_{11}=1$ we can make all elements zero below the element $y$ in the 1-st column. So we obtain the indecomposable direct summand of $\mathbf{T}$ of the form (1). So we can assume $v_{1}(y)=n<0$.

Therefore $y^{-1} \in O_{1}$. By the row transformations on $\mathbf{T}_{3}$ we can make all elements zero below the element $y$ in the 1 -st column. Since $y^{-1} \in O_{1}$ we can add the $k$-th row of $\mathbf{T}$ to the first row of it. Then we obtain in the reducing matrix the element $a_{11}=0$ and $v_{1}\left(a_{1 j}\right)>0$ for all $j>1$ since $v_{1}\left(y^{-1} t_{k j}\right) \geq 0$ owing $v_{1}(y)$ is minimal. Then using the elements $a_{i i}=1$ for $i=2, \ldots, k-1$ we reduce our matrix to the following form:

$$
\mathbf{T}=\left[\begin{array}{ll}
\mathbf{X} & \mathbf{O} \\
\mathbf{Y} & \mathbf{O}
\end{array}\right] \text {, where } \mathbf{T}=\left[\begin{array}{ll}
\mathbf{X} & \mathbf{O} \\
\mathbf{Y} & \mathbf{O}
\end{array}\right] \text { and } \mathbf{Y}=\left[\begin{array}{cc}
y & * \\
\mathbf{O} & *
\end{array}\right] .
$$

Since element $y \in D$ can be represented in the form $y=a b$, where $a \in O_{1}{ }^{*}$, $b \in O_{2}{ }^{*}$, then multiplying the 1 -st column by $b^{-1}$ and the $k$-th row by $a^{-1}$ we can make the element $t_{k 1}$ to be 1 , i.e. we obtain:

$$
\mathbf{T}=\left[\begin{array}{ll}
\mathbf{X} & \mathbf{O} \\
\mathbf{S} & \mathbf{O}
\end{array}\right], \text { where } \mathbf{S}=\left[\begin{array}{rr}
1 & * \\
\mathbf{O} & \mathbf{M}_{3}
\end{array}\right]
$$

Now all elements in the $k$-th row we can make 1 , since $O_{1}+O_{2}=D$. So we obtain the decomposition of matrix $\mathbf{T}$ :

$$
\mathbf{T}=\left[\begin{array}{ll}
1 & \mathbf{O} \\
\mathbf{O} & \mathbf{A}
\end{array}\right], \text { where } \mathbf{A}=\left[\begin{array}{cc}
\mathbf{I} & \mathbf{O} \\
\mathbf{M}_{3} & \mathbf{O}
\end{array}\right]
$$


Since the dimension of the matrix $\mathbf{M}_{3}$ is smaller than the previous matrix, by mathematical induction we obtain that the matrix $\mathbf{T}$ can be reduced to the form (11), as required.

\section{Matrix problem II}

Let $O_{1}, O_{2}$ be a distinct discrete valuation rings of the common division ring of fractions $D$. Given a matrix $\mathbf{T}$ with entries in $D$ with the following admissible transformations:

1) left $O_{1} \cap O_{2}$ - elementary transformations of rows of the matrix $\mathbf{T}$.

2) right $D$ - elementary transformations of columns of the matrix $\mathbf{T}$.

Find all indecomposable matrices.

Lemma 4.2. The matrix problem II is of unbounded representation type.

\section{Proof.}

By proposition 3.8 there exists an element $x \in D$ such that $x^{n} \notin A$ and $x^{-n} \notin A$ for any $n \in \mathbf{N}$, where $A=O_{1} \cap O_{2}$.

Then the matrix of the following form:

$$
\mathbf{T}=\left[\begin{array}{cccc}
1 & 0 & \cdots & 0 \\
0 & 1 & \cdots & 0 \\
\vdots & \vdots & \ddots & \vdots \\
0 & 0 & \cdots & 1 \\
x & x^{2} & \cdots & x^{n}
\end{array}\right]
$$

is indecomposable under admissible transformations 1) and 2) of the matrix problem II for any $n \in \mathbf{N}$.

\section{Matrix problem III}

Let $O_{1}, O_{2}$ be distinct discrete valuation rings of the division ring $D$. Given a matrix $\mathbf{T}$ with entries in $D$ partitioned into two vertical strips:

$$
\mathbf{T}=\left[\begin{array}{l|l}
\mathbf{A}_{1} \mid \mathbf{A}_{2}
\end{array}\right]
$$

with the following admissible transformations:

1) $O_{1}$ - elementary transformations of rows of the matrix $\mathbf{T}$.

2) $\mathrm{O}_{2}$ - elementary transformations of columns of the matrix $\mathbf{A}_{1}$.

3) $D$ - elementary transformations of columns of the matrix $\mathbf{A}_{2}$.

Find all indecomposable matrices. 
Lemma 4.3. The matrix problem III is of the unbounded representation type.

Proof. By admissible transformations we can reduce our matrix problem to the form:

$$
\mathbf{T}=\left[\begin{array}{l|l|l|l}
\mathbf{I} & \mathbf{O} & \mathbf{O} & \mathbf{B} \\
\mathbf{O} & \mathbf{O} & \mathbf{I} & \mathbf{O} \\
\mathbf{O} & \mathbf{O} & \mathbf{O} & \mathbf{O}
\end{array}\right]
$$

where there are possible the following admissible transformations:

1) left $O_{1} \cap O_{2}$ - elementary transformations of rows of the matrix $\mathbf{B}$;

2) right $D$ - elementary transformations of columns of the matrix $\mathbf{B}$.

Therefore we get the matrix problem II which is unbounded representation type by Lemma 4.2.

\section{Conclusions}

In this article we consider the approximation theorem for discrete valuation rings and obtain some important corollaries from this theorem, which gives the possibility to solve mixed flat matrix problems over several district valuation rings with common skew field of fractions. We give the solution of some such mixed flat matrix problems.

\section{References}

[1] Zavadskij A.G., Revitskaya U.S., A matrix problem over a discrete valuation rings, Mat. Sb. 1999, 190, 6, 835-858.

[2] Zavadskij A.G., Kirichenko V.V., Torsion-free modules over primary rings, Zap. Nauchn. Sem. LOMI 1976, 57, 100-116 (in Rus.); English transl. in J. Soviet Math. 1979, 11.

[3] Zavadskij A.G., Kirichenko V.V., Semimaximal rings of finite type, Mat. Sb. 1977, 103, 323-345.

[4] Gubareni N.M., Semiperfect right hereditary rings of module representation type, Preprint 78.1, Academy of Sciences of USSR, IM, Kiev 1978 (in Rus.).

[5] Kirichenko V.V., Kostyukovich P.P., Yaremenko Yu.V., Biserial rings and modules over these rings, Algebraic structures and their applications, Acadamy of Sciences of USSR, IM, Kiev 1988, 43-74 (in Rus.).

[6] Hazewinkel M., Gubareni N., Kirichenko V.V., Algebras, Rings and Modules. Vol. 1, Kluwer Academic Publisher, 2004.

[7] Hazewinkel M., Gubareni N., Kirichenko V.V., Algebras, Rings and Modules. Vol. 2, Springer, 2007.

[8] Schilling O.F.G., Noncommutative valuations, Bull. Amer. Math. Soc. 1945, 51, 297-304.

[9] Krull W., Allgemeine Bewertungstheorie, J. Reine Angew. Math. 1932, 167, 160-196. 
[10] Gubareni N., Valuation and discrete valuation rings, Scientific Research of the Institute of Mathematics and Computer Science 2011, 1(10), 61-70.

[11] Brungs H.H., Gräter J., Noncommutative Prüfer and valuation rings, Comtemporary Mathematics, 1992, 131, Part 2, 253-269.

[12] Gräter J., Lokalinvariant Bewertungen, Math. Z. 1986, 192, 183-194.

[13] Brungs H.H., Gräter J., Valuation rings in finite-dimensional division algebras, J. Algebra 1989, 120, 90-99.

[14] Mathiak K., Valuations of Skew Fields and Projective Hjelmslev Spaces, Springer-Verlag, 1986. 